\title{
Real-Time Pose Detection for Magnetic Medical Devices
}

\author{
Christian Di Natali, Marco Beccani, and Pietro Valdastri \\ STORM Lab, Department of Mechanical Engineering, Vanderbilt University, Nashville, TN 37235 USA
}

\begin{abstract}
Magnetic coupling is one of the few physical phenomena capable of transmitting motion across a physical barrier. In gastrointestinal endoscopy, remote magnetic manipulation has the potential to make screening less invasive and more acceptable, thus saving lives by early diagnoses and treatment. Closed-loop control of the magnetic device position is crucial for a safe and reliable operation. In order to implement closed-loop control, the pose (position and orientation) of the device must be available in real-time. This becomes challenging if magnetic coupling is achieved by permanent magnets, since the strong magnetic field required for manipulation interferes with current localization techniques. In this work, we present a novel real-time pose detection strategy that is compatible with magnetic manipulation based on permanent magnets. The localization algorithm combines multiple sensor readings with a pre-calculated magnetic field map. The proposed approach is able to provide an average error below $5 \mathrm{~mm}$ in position detection, and below $19^{\circ}$ for angular motion within a spherical workspace of $15 \mathrm{~cm}$ in radius.
\end{abstract}

Index Terms-Biomedical equipment, gastrointestinal endoscopy, magnetic medical devices, pose detection, wireless capsule endoscopy.

\section{INTRODUCTION}

$\mathbf{T}$ HE gastrointestinal (GI) tract is home to some of the most deadly human diseases. Today, flexible endoscopy allows for effective diagnosis and treatment through its different districts. However, this technique is not well perceived by patients due to the pain associated with the procedure and the frequent need for sedation. A more acceptable technique is wireless capsule endoscopy (WCE), introduced for the first time in 2000 and considered today the gold standard for diagnosis of small intestine diseases [1]. WCE entails the ingestion of a miniature pill-size camera that moves passively through the digestive system and enables visualization of the bowel without the need for intubation, insufflation, or sedation, thus offering an appealing alternative to flexible endoscopy.

Remote manipulation of capsule endoscopes has the potential to extend the reach of WCE to other GI districts, allowing the physician to have direct control of the camera viewpoint [1]. Among the different approaches proposed to achieve active locomotion, the use of magnetic fields to control the capsule pose (i.e., position and orientation) has the advantage of preventing the need for onboard actuators, dedicated mechanisms, and energy. This approach is currently being pursued by the major commercial players in the field of GI endoscopy (i.e., Olympus and Siemens in [2] and Given Imaging in [3]) and by several research group worldwide [1], [4]-[8]. To achieve closed-loop control, the pose of the remote device must be determined in real-time and used in conjunction with the desired location or direction of motion specified by the user. Most of the localization techniques developed to date-mainly designed to track the capsule endoscope as it passively travels in the GI tract — are not compatible with magnetic manipulation due to electromagnetic interference [7]. A high-frequency alternating magnetic field, generated by pairs of Cartesian coils, can be used in conjunction with a resonating coil [9] or Hall Effect sensors [10] em-

\footnotetext{
Manuscript received October 31, 2012; accepted January 10, 2013. Date of current version July 15, 2013. Corresponding author: P. Valdastri (e-mail: p.valdastri@vanderbilt.edu).

Color versions of one or more of the figures in this paper are available online at http://ieeexplore.ieee.org.

Digital Object Identifier 10.1109/TMAG.2013.2240899
}

bedded in the capsule for localization during magnetic manipulation. This approach can provide sub-millimeter resolution, but is limited to three degrees of freedom (DOF).

A different strategy to achieve magnetic coupling is based on permanent magnets [4], [11], [12]. Compared to coils, permanent magnets can generate higher fields in a smaller form factor. In this case, the capsule can be localized by measuring the magnetic field generated by the external permanent magnet (EPM) at the capsule, taking advantage of onboard sensors. This approach is adopted in [12] to achieve capsule localization in 5 DOF with an accuracy of $15 \mathrm{~mm}$. Inertial sensing is used in conjunction with Hall effect sensors to localize an endoscopic capsule driven by a robotic EPM in [13]. This method achieves a $10 \mathrm{~mm}$ resolution on $3 \mathrm{DOF}$.

In this paper we describe a novel approach to detect in realtime the 6-DOF pose of a magnetic capsule endoscope. The proposed method takes advantage of the external magnetic field generated for manipulation, allowing for actuation and localization at the same time. This method is optimized for a cylindrical EPM with axial magnetization, but can be generalized straightforwardly to any external source of magnetic field.

\section{MATERIALS}

\section{A. Principle of Operation}

The proposed method takes advantage of measuring the magnetic field generated by the EPM at the two ends of the capsule. Two Cartesian triplets of Hall Effect sensors are used to acquire the three components of the field (i.e., $\left.B_{x 1}^{\prime}, B_{y 1}^{\prime}, B_{z 1}^{\prime}, B_{x 2}^{\prime}, B_{y 2}^{\prime}, B_{z 2}^{\prime}\right)$. The resulting $\overrightarrow{B_{1}^{\prime}}$ and $\overrightarrow{B_{2}^{\prime}}$ vectors are referred to the center of the capsule $\left(\left[x^{\prime}, y^{\prime}, z^{\prime}\right]\right.$ reference frame represented in Fig. 1). The inclination of the capsule (i.e., $\alpha^{\prime}$ and $\beta^{\prime}$ defined as pitch and yaw of $\left[x^{\prime}, y^{\prime}, z^{\prime}\right]$ with respect to the world reference frame $\left.\left[x_{w}, y_{w}, z_{w}\right]\right)$ is also recorded in real-time with an onboard triaxial accelerometer. Given these 8 real-time measurements, the proposed algorithm is able to provide the pose of the capsule in terms of the distance vector $\vec{d}$ and the three Euler's orientation angles $(\alpha, \beta, \gamma)$ referred to a Cartesian frame $[x, y, z]$ located at the center of the EPM. In case the EPM arrangement is known with 


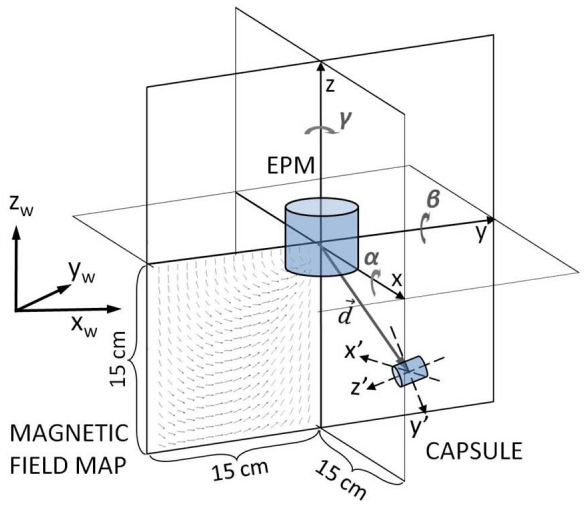

Fig. 1. Schematic view of the EPM and the capsule with the different reference frames used in the algorithm. A slice of the magnetic field map is represented in the lower left quadrant of the workspace.

respect to the world frame, the transformation from $[x, y, z]$ to $\left[x_{w}, y_{w}, z_{w}\right]$ (and viceversa) can be easily obtained using the real-time data acquired from the robot encoders.

The method we developed is based on an iterative algorithm written in Matlab (Mathworks, Inc., USA). The first step consists in the calibration of the initial roll angle $\gamma_{0}$ as baseline. This is required because the roll $\gamma$ is the only orientation angle that cannot be measured by the triaxial accelerometer. This calibration requires positioning the capsule with a known orientation for $\gamma$ with respect to the EPM.

The next steps are to acquire $\overrightarrow{B_{1}^{\prime}}$ and $\overrightarrow{B_{2}^{\prime}}$ from the onboard Hall Effect sensors, $\alpha^{\prime}$ and $\beta^{\prime}$ from the accelerometer, and to express the magnetic field vectors with respect to $[x, y, z]$ by the following transformation:

$$
\overrightarrow{B_{i}}=R^{w} R_{\prime}^{w} R_{w}^{T} \overrightarrow{B_{i}^{\prime}} \quad \text { with } i=1,2
$$

where $R^{w}$ and its transpose $R_{w}^{T}$ build the similarity transformation converting coordinates from $\left[x_{w}, y_{w}, z_{w}\right]$ to $[x, y, z]$, while the $3 \times 3$ rotation matrix $R_{w}^{\prime}$, shown in the first equation below, where $c(\cdot)$ and $s(\cdot)$ are cosine and sine trigonometric functions, $\alpha^{\prime}$ and $\beta^{\prime}$ are the pitch and yaw acquired by the triaxial accelerometer, while $\gamma$ is equal to $\gamma_{0}$ for the first iteration. The angle $\gamma$ is then generated by the algorithm for the following iterations, as detailed later in the paper.

The values of the magnetic field at the capsule are then used in conjunction with a pre-calculated numerical map of the magnetic field generated by the EPM. In this map, the magnetic field vectors are associated with the distance vector $\vec{d}$. A search is performed in the map to find the values of $\left(d_{x}, d_{y}, d_{z}\right)$ related to the magnetic field vectors that are the closest to the measured values $\overrightarrow{B_{1}}$ and $\overrightarrow{B_{2}}$. The computational time and the precision of the proposed method strongly depend on the search function and the magnetic field map. In case an axially magnetized cylindrical permanent magnet is used as EPM, the magnetic field can be expressed in cylindrical coordinates. Therefore, the algorithm derives

$$
\left\{\begin{array}{l}
\overrightarrow{B_{i}^{c}}=B_{r i} \hat{r}+B_{z i} \hat{z}=\sqrt{\left(B_{x i}^{2}+B_{y i}^{2}\right)} \hat{r}+B_{z i} \hat{z} \\
\theta_{i}=\arctan \left(\frac{B_{y i}}{B_{x i}}\right)
\end{array}\right.
$$

for $i=1,2$, where $B_{r i} \hat{r}$ is the radial component and $B_{z i} \hat{z}$ is axial component of $\overrightarrow{B_{i}^{c}}$. The azimuthal angle of the cylindrical coordinates is expressed by $\theta_{i}$.

Magnetic models based upon the theories and the methods used in the analysis of steady currents, permanent magnets, and magnetic circuits [14], [15] are applied to generate the field density map to cover a $30 \mathrm{~cm} \times 30 \mathrm{~cm} \times 30 \mathrm{~cm}$ workspace centered on the EPM. In particular, the Magnetic Current Model (MCM) allows for a numerical solution by finite element integration. The MCM for an axially magnetized cylindrical magnet is expressed as follows

$$
\overrightarrow{B(p)}=\frac{\mu_{0}}{4 \pi} \oint_{S^{\prime \prime}} j_{m}\left(p^{\prime \prime}\right) \times \frac{\left(p-p^{\prime \prime}\right)}{\left|p-p^{\prime \prime}\right|^{3}} d s^{\prime \prime}
$$

where $p$ indicates a generic point belonging to the workspace, $p^{\prime \prime}$ is a point on the magnet surface, $j_{m}$ is the equivalent surface current density, $S^{\prime \prime}$ is the EPM integration surface, and $\mu_{0}$ is the vacuum permeability constant. Expressing (3) in cylindrical coordinates, the magnetic field becomes (4), shown at the bottom of the page, where $r, \theta$, and $z$ are the cylindrical coordinates of the generic point $p$, while $R$ and $L$ are the radius and the height of the EPM, respectively. The constant $K=\left(\mu_{0} M_{s}\right) /(4 \pi)$ accounts for the EPM magnetic remanence $M_{s}$.

Thanks to the cylindrical symmetry of the selected EPM, the value of $\overrightarrow{B^{c}}$ is only a function of $r$ and $z$, since its $\theta$ component is zero (see (4)). This allows to simplify the magnetic field map to a single $15 \mathrm{~cm} \times 30 \mathrm{~cm}$ slice. Also, since we can assume the capsule to be inside the body of a patient laying in the $z<0$ space, the slice can be further reduced to a $15 \mathrm{~cm} \times 15 \mathrm{~cm}$ domain, as represented in Fig. 1. Each point belonging to this slice is expressed as a two component magnetic field vector $\overrightarrow{B^{c}(p)}=B_{r}(p) \hat{r}+B_{z}(p) \hat{z}$ with a univocal association to a point $p$ in the domain. The values of the bi-dimensional map are calculated by applying (4) to the specific EPM used in our

$$
R_{w}^{\prime}=\left[\begin{array}{ccc}
c\left(\beta^{\prime}\right) c(\gamma) & c(\gamma) s\left(\alpha^{\prime}\right) s\left(\beta^{\prime}\right)-c\left(\alpha^{\prime}\right) s(\gamma) & s\left(\alpha^{\prime}\right) s(\gamma)+c\left(\alpha^{\prime}\right) c(\gamma) s\left(\beta^{\prime}\right) \\
c\left(\beta^{\prime}\right) s(\gamma) & c\left(\alpha^{\prime}\right) c(\gamma)-s(\gamma) s\left(\alpha^{\prime}\right) s\left(\beta^{\prime}\right) & s(\gamma) c\left(\alpha^{\prime}\right) s\left(\beta^{\prime}\right)-s\left(\alpha^{\prime}\right) c(\gamma) \\
-s\left(\beta^{\prime}\right) & c\left(\beta^{\prime}\right) s\left(\alpha^{\prime}\right) & c\left(\alpha^{\prime}\right) c\left(\beta^{\prime}\right)
\end{array}\right]
$$$$
\overrightarrow{B^{c}(p)}=K \int_{L}^{0} \int_{0}^{2 \pi} \frac{(R-r) \hat{z}+\left(z-z^{\prime}\right) \hat{r}}{\left.\mid R^{2}+r^{2}-2 r R c\left(\theta-\theta^{\prime}\right)+\left(z-z^{\prime}\right)^{2}\right)\left.\right|^{\frac{3}{2}}} d \theta^{\prime} d z^{\prime}
$$ 


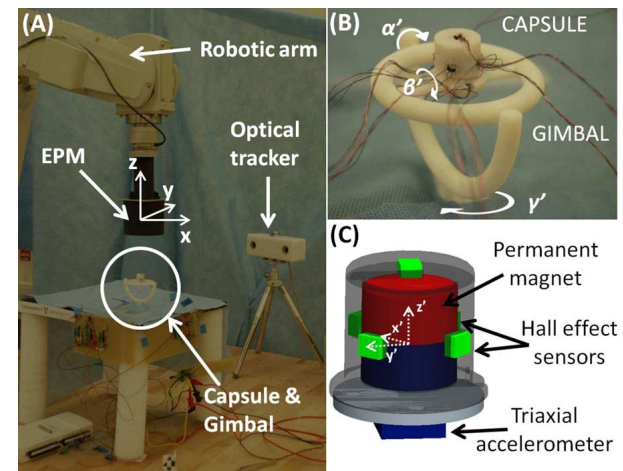

Fig. 2. Validation of the proposed pose detection method: (A) View of the platform; (B) Close view of the capsule and the gimbal; (C) Schematic view of the capsule.

platform with a spatial resolution of $0.2 \mathrm{~mm}$. Of course, the resolution of the map is chosen as a trade-off between computational time and localization accuracy.

A sequential search is performed into the map to find $\left(d_{r i}, d_{z i}\right)$ associated with the measured $\left(B_{r i}, B_{z i}\right)$ for $i=1,2$. The third cylindrical coordinate $d_{\theta i}$ can be calculated from the values of $B_{y i}$ and $B_{x i}$ by applying (2). The position of the capsule center is then obtained by averaging $\overrightarrow{d_{1}}$ and $\overrightarrow{d_{2}}$. The roll $\gamma$ is achieved as the angle in between the $x$ axis and the line intercepting the projections of $\overrightarrow{d_{1}}$ and $\overrightarrow{d_{2}}$ on the horizontal plane. At this point, the capsule pose is fully identified with respect to $[x, y, z]$ and the algorithm restart from acquiring $\overrightarrow{B_{1}^{\prime}}, \overrightarrow{B_{2}^{\prime}}, \alpha^{\prime}$, and $\beta^{\prime}$, this time adopting the actual $\gamma$ in computing the $R_{w}$ matrix in (1).

\section{B. Platform Overview}

The proposed method for capsule pose detection was assessed using the experimental platform represented in Fig. 2(A). The platform consists of a robotic arm holding the EPM, and a capsule embedded with a permanent magnet and the localization module. The capsule is mounted on a gimbal (Fig. 2(B)), that allows 3 DOF rotations. Since the capsule is designed to operate inside the human body, thus in a loose environment, the gimbal allows to validate the algorithm without constraining the sensing module in a fixed orientation. An optical tracker (Micron Tracker, Claron Technology, Inc., USA) is used as reference system to measure the angular motion of the capsule.

The robotic arm (RV6SDL, Mitsubishi, Inc., Japan) has 6 DOF and a resolution of motion of $1 \mu \mathrm{m}$. The EPM is an off-theshell cylindrical permanent magnet in $\mathrm{NdFeB}$, with $\mathrm{N} 52$ axial magnetization, a remanance of $1.48 \mathrm{~T}$, a diameter of $5 \mathrm{~cm}$ and a height of $5 \mathrm{~cm}$ (K\&J Magnetics, Inc., USA). The permanent magnet embedded in the capsule has the same features as the $\mathrm{EPM}$, but is $11 \mathrm{~mm}$ in diameter, and $11 \mathrm{~mm}$ in height. The magnetic coupling between the two magnets allows for an effective manipulation, as reported in [16].

The localization module is designed all around the onboard magnet in order to be easily integrated in a device operated by magnetic manipulation. The module consists of a digital triaxial accelerometer (LIS331DLH, STM, Switzerland) and six uniaxial Hall effect sensors (CYP15A, Chen Yang Technologies $\mathrm{GmbH} \& \mathrm{Co} . \mathrm{KG}$, Germany). The Hall Effect sensors were chosen with a large dynamic range (i.e., from $0.1 \mu \mathrm{T}$ to $2 \mathrm{~T}$ ) to accommodate the offset due to the internal magnet (i.e., about $10 \%$ of the dynamic range). As represented in Fig. 2(C), the Hall Effect sensors were positioned along $\left[x^{\prime}, y^{\prime}, z^{\prime}\right]$ at the opposite sides of the capsule in order to maximize the separation between $\overrightarrow{B_{1}^{\prime}}$ and $\overrightarrow{B_{2}^{\prime}}$ readings. The separation distance for a couple of Hall Effect sensors laying on the same axis is $12 \mathrm{~mm}$. The overall size of the localization module and the onboard magnet is $15 \mathrm{~mm}$ in diameter and $18 \mathrm{~mm}$ in height. Both gimbal and capsule were fabricated by rapid prototyping (OBJET 30, Objet Ltd., Israel). Data are acquired from the Hall effect sensors and the accelerometer by an USB data acquisition board (DAQ 6211, National Instrument, Inc., USA) with a 16-bit resolution at each iteration of the algorithm. The sensor size, power consumption, and data output are compatible with wireless operation. In particular, a wireless microcontroller (e.g., CC2530, Texas Instruments, Inc., USA) and a high resolution analog to digital converter (e.g., ADS8320, Texas Instrument, Inc., USA) can be used to acquire the measurements at the same sampling rate and resolution, and send them to an external receiver by radiofrequency communication [16]. The platform is controlled by a software written in $\mathrm{C}++$ and communicates in real-time with the data acquisition board and the robotic arm controller.

\section{EXPERIMENTAL VALIDATION AND RESULTS}

In order to validate the proposed method, the robotic arm was programmed to move the EPM within the $z<0$ portion of the workspace represented in Fig. 1. Considering the capsule fixed to the gimbal at $[x=0 \mathrm{~cm}, y=0 \mathrm{~cm}, z=-15 \mathrm{~cm}]$, the robot scanned a $30 \mathrm{~cm} \times 30 \mathrm{~cm}$ area on the $(x, y)$ plane at nine different vertical positions (i.e., $z$ varying from $-8 \mathrm{~cm}$ to $0 \mathrm{~cm}$ in $1-\mathrm{cm}$ steps). The benchmark for validation was provided by the robotic arm encoders (resolution of $1 \mu \mathrm{m}$ ) for $\vec{d}$, and by the optical tracker (resolution of $0.3^{\circ}$ ) for $\alpha, \beta$, and $\gamma$.

Since the proposed method is based on magnetic field measurement, the error is expected to increase with the separation distance between the source of magnetic field and the position where it is measured. Therefore, the error in detecting capsule pose is reported in Fig. 3 for three different values of $|\vec{d}|$ (i.e., $10 \mathrm{~cm}, 12.5 \mathrm{~cm}$, and $15 \mathrm{~cm}$ ) within a typical range of operation for magnetic capsule endoscopes [11]. In particular, if the capsule is within $10 \mathrm{~cm}$ from the EPM, the average error and the standard deviation are $-4.3 \pm 2.1 \mathrm{~mm}$ for $x,-4.5 \pm 1.9 \mathrm{~mm}$ for $y,-3.9 \pm 1.8 \mathrm{~mm}$ for $z$, and $-12^{\circ} \pm 29^{\circ}$ for $\gamma$. In case the capsule is within $15 \mathrm{~cm}$ from the EPM, the average error remains in the same range, but the standard deviation significantly increases. The error and standard deviation for $|\vec{d}|<15 \mathrm{~cm}$ are $-3.4 \pm 3.2 \mathrm{~mm}$ for $x,-3.8 \pm 6.2 \mathrm{~mm}$ for $y, 3.4 \pm 7.3 \mathrm{~mm}$ for $z$, and $-19^{\circ} \pm 50^{\circ}$ for $\gamma$ if the capsule is within $15 \mathrm{~cm}$ from the EPM. The angles $\alpha$ and $\beta$ are measured by the accelerometer, therefore their error is not dependent on $|\vec{d}|$. The average error and the standard deviation for $\alpha$ and $\beta$ are $-6^{\circ} \pm 18^{\circ}$ and $3^{\circ} \pm 20^{\circ}$, respectively.

For a more straightforward interpretation of results, it is possible to define the event of successful localization. A localization is successful if the capsule estimated position $\vec{d}$ is falling 

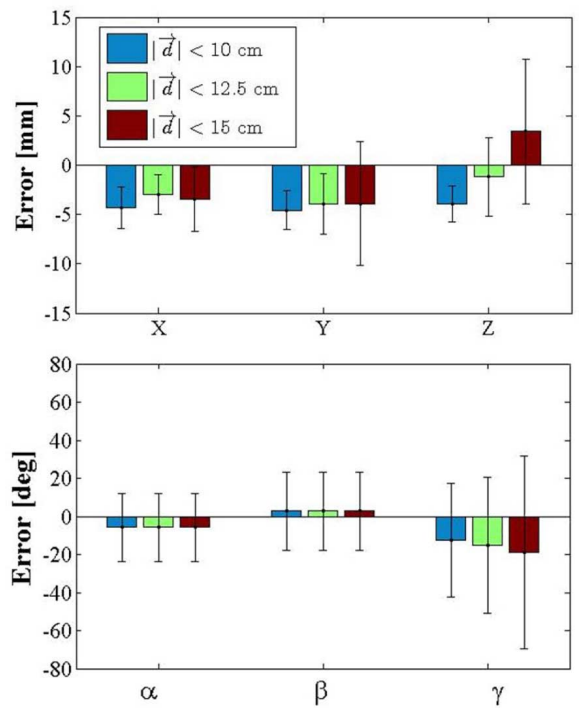

Fig. 3. Error bar plot for the proposed pose detection method in the six DOF, reported for three different values of $|\vec{d}|$. The bar height represents the average error, while the whiskers account for standard deviation.

TABLE I

LOCALIZATION SUCCESS RATE FOR DIFFERENT $|\vec{d}|$

\begin{tabular}{c||c|c|c}
\hline & $|\vec{d}|<10 \mathrm{~cm}$ & $|\vec{d}|<12.5 \mathrm{~cm}$ & $|\vec{d}|<15 \mathrm{~cm}$ \\
\hline \hline $\begin{array}{c}\text { Success } \\
\text { Rate }\end{array}$ & $82 \%$ & $66 \%$ & $48 \%$ \\
\hline $\begin{array}{c}\text { Total } \\
\text { Measurements }\end{array}$ & 4,000 & 12,500 & 26,000 \\
\hline
\end{tabular}

within a spherical volume centered in the capsule actual position and having a diameter of $11 \mathrm{~mm}$, as current capsule endoscopes [1]. The success rates of the proposed algorithm related to the total number of measurements are reported in Table I as a function of $|\vec{d}|$. The full range of magnetic field at the capsule is within $\pm 120 \mathrm{mT}$, while typical values for the considered application are in the range of $\pm 50 \mathrm{mT}$.

As regards computational time, a single instance of the algorithm takes $14 \mathrm{~ms}$, while the search requires an average of $5 \mathrm{~ms}$. These results are compatible with the closed-loop control cycle of the robotic arm controlling the EPM (i.e., typically $20 \mathrm{~ms}$ ).

\section{CONCLUSION}

In this paper we propose a novel approach to detect in realtime the 6-DOF pose of a magnetic capsule endoscope, combining multiple sensor readings with pre-calculated magnetic field values. This solution is compatible with magnetic manipulation and is optimized for a cylindrical EPM with axial magnetization. The sensing module is designed for wireless operation, and the algorithm has a computational time that allows real-time teleoperation. The proposed approach is able to provide an average error below $5 \mathrm{~mm}$ in position detection, and below $19^{\circ}$ for angular motion within a spherical workspace of $15 \mathrm{~cm}$ in radius, centered on the EPM.

Future works will aim to improve pose detection accuracy by real-time filtering. In particular, Kalman or particle filters may be a viable option. The localization module will be integrated in a wireless capsule. Then, the real-time pose detection algorithm will be used to achieve closed-loop control of capsule position by magnetic manipulation.

\section{ACKNOWLEDGMENT}

This work was supported by the National Science Foundation under Grant No. CNS-1239355 and by the National Center for Advancing Translational Sciences under Grant No. UL1TR000445-06. The authors wish to thank G. Bassani for helping with the magnetic field map and N. Simaan for lending the optical tracker.

\section{REFERENCES}

[1] P. Valdastri, M. Simi, and R. J. Webster, III, "Advanced technologies for gastrointestinal endoscopy," Аnnu. Rev. Biomed. Engrg., vol. 14, pp. 397-429, 2012.

[2] H. Keller, A. Juloski, H. Kawano, M. Bechtold, A. Kimura, H. Takizawa, and R. Kuth, "Method for navigation and control of a magnetically guided capsule endoscope in the human stomach," in Proc. IEEE Int. Conf. on Biomedical Robotics and Biomechatronics (BioRob), Jun. 2012, pp. 859-865.

[3] J. Keller, C. Fibbe, C. Volke, J. Gerber, A. C. Mosse, M. ReimannZawadzki, E. Rabinovitz, P. Layer, D. Schmitt, V. Andresen, U. Rosien, and P. Swain, "Inspection of the human stomach using remote-controlled capsule endoscopy: A feasibility study in healthy volunteers (with videos)," Gastro. Endosc., vol. 73, no. 1, pp. 22-28, 2011.

[4] A. W. Mahoney and J. J. Abbott, "Control of untethered magnetically actuated tools with localization uncertainty using a rotating permanent magnet," in Proc. IEEE Int. Conf. on Biomedical Robotics and Biomechatronics (BioRob), Jun. 2012, pp. 1632-1637.

[5] M. Gao, C. Hu, Z. Chen, H. Zhang, and S. Liu, "Design and fabrication of a magnetic propulsion system for self-propelled capsule endoscope," IEEE Trans. Bio-Med. Eng., vol. 57, no. 12, pp. 2891-2902, 2010.

[6] X. Wang, M. Q. H. Meng, and X. Chen, "A locomotion mechanism with external magnetic guidance for active capsule endoscope," in Proc. Annu. Int. Conf. IEEE Engineering in Medicine and Biology Society, Jan. 2010, pp. 4375-4378.

[7] T. D. Than, G. Alici, H. Zhou, and W. Li, "A review of localization systems for robotic endoscopic capsules," IEEE Trans. Bio-Med. Engrg., vol. 59, no. 9, pp. 2387-2399, 2012.

[8] Northern Digital Inc. [Online]. Available: www.ndigital.com/medical/ aurora.php, 2005

[9] S. I. Aoki, Y. Uchiyama, S. Arai, S. Ishiyama, and S. Yabukami, "Detecting System of Position and Posture of Capsule Medical Devices," U.S. Patent US-2005/1216231 A1, 2005.

[10] K. M. Miller, A. W. Mahoney, T. Schmid, and J. J. Abbott, "Proprioceptive magnetic-field sensing for closed-loop control of magnetic capsule endoscopes," in IEEE Int. Conf. Intelligent Robots and Systems (IROS), Oct. 2012, pp. 1994-1999.

[11] G. Ciuti, P. Valdastri, A. Menciassi, and P. Dario, "Robotic magnetic steering and locomotion of capsule endoscope for diagnostic and surgical endoluminal procedures," Robotica, vol. 28, no. 2, pp. 199-207, 2009.

[12] M.-G. Kim, Y.-S. Hong, and E.-J. Lim, "Position and orientation detection of capsule endoscopes in spiral motion," Int. J. Precision Engrg. Mfg., vol. 11, no. 1, pp. 31-37, 2010.

[13] M. Salerno, F. Mulana, R. Rizzo, A. Landi, and A. Menciassi, "Magnetic and inertial sensor fusion for the localization endoluminal diagnostic devices," Int. J. Comput. Assist. Radiol. Surgery (CARS), vol. 7, no. S1, pp. 229-235, May 2012.

[14] E. P. Furlani, Permanent Magnet and Electromechanical Devices. Amsterdam, The Netherlands: Elsevier, 2001, pp. 131-135.

[15] E. Durand, Electrostatique. France: Masson Editeur, 1964, vol. 1, pp. 248-251.

[16] M. Beccani, C. Di Natali, M. E. Rentschler, and P. Valdastri, "Wireless tissue palpation: Proof of concept for a single degree of freedom," presented at the IEEE Int. Conf. on Robotics and Automation (ICRA), Karlsruhe, Germany, May 6-10, 2013. 